\title{
LA INTERNACIONALIZACIÓN DE LA EDUCACIÓN SUPERIOR
}

\section{Cambio Institucional en las Universidades Públicas de la Región Noroeste de México}

Internationalization of Higher Education: Institutional Change in the Public Universities of the Northwest Region of Mexico

Alma Beatriz Navarro Cerda

Universidad Autónoma de Baja California, México

\section{KEY WORDS}

MT Educacional

Administration

NT3 University

Cooperation

TR

Exchange Programs

\section{ABSTRACT}

Internationalization in higher education has emerged as a parallel to the need to conceptualize globalization. It is conceived and defined based on the support of various disciplines within the social sciences, such as economics and political science, and a common definition of internationalization in higher education is still lacking. In this paper, a qualitative methodology is used to emphasize the pragmatic aspects of the internationalization of higher education in the public universities of Mexico belonging to the northwest region in terms of institutional change.
PALABRAS CLAVE

MT Administración de la

Educación

TE3 Cooperación

Universitaria TR Programas

de Intercambio

\section{RESUMEN}

La internacionalización en la educación superior ha surgido como un elemento paralelo a la necesidad de conceptualizar a la globalización. Se concibe y se define a partir del apoyo de diversas disciplinas dentro del ámbito de las ciencias sociales, tales como la economía y la ciencia política, haciendo aun falta una definición en comun sobre la internacionalización en la educación superior. En este trabajo se utiliza una metodología cualitativa, para hacer énfasis en los aspectos pragmáticos de la internacionalización de la educación superior en las Universidades Públicas de México pertenecientes a la región Noroeste en términos cambio institucional. 


\section{La internacionalización de la Educación Superior como concepto y como política}

$\mathrm{L}$ as diversas aportaciones conceptuales en educación superior y su relación con la globalización son resultado de los enfoques disciplinarios que han sido utilizados para explicar y contextualizar este fenómeno de acuerdo a la multiplicidad de escenarios y realidades que se presentan o que son percibidos. Aunado a ello la integración del elemento de la internacionalización en la educación superior ha contribuido en la diversidad de conceptos y definiciones en las cuales, el eje que tienen en común es "la globalización”.

De ahí la necesidad de hacer mención que la globalización se entenderá en este trabajo, como el fenómeno que ha suscitado los cambios drásticos en torno a las formas de comunicación, eliminando de manera gradual el espacio y tiempo en las interconexiones (Held, 2000), alrededor del mundo, los cuales se han combinado en cambios económicos, sociales y culturales (OECD, 2009). Es por ello, que a través del impulso de la globalización se han observado cambios contundentes en el contexto internacional teniendo efectos sobre la educación superior (Nayyar, 2008). El cual como ya se mencionó ha sido apoyado por la evolución y el uso de las tecnologías de la información. Por lo tanto, se entiende que estos cambios en materia educativa son motivados por la globalización, lo cual ha generado que se perciba de forma diferente el propio concepto de educación, así como la necesidad que ha tenido de replantearse en ¿qué papel que juega el docente dentro del proceso?.

Vinculado con la globalización se ha percibido como fenómeno y construcción del concepto a la "internacionalización", el cual en materia de educación superior se asume como una estrategia para hacer frente a la globalización, en donde las universidades actúan en contextos diferentes asociados a su capacidad de asimilación de los procesos globales (Knight, 2005). También es importante aclarar que existen diversas aportaciones de internacionalización que contienen elementos vinculados con aspectos sociales, los cuales en términos de política en México pueden ser asociados principalmente como un elemento de "calidad" por considerarse, una característica que debe ser contenida en las políticas educativas en torno a la demanda y presiones sociales.

Es así que la principal relación entre la globalización y la internacionalización es que se tiene como sustento y unidad de vínculo a la "educación superior". En ese sentido el debate teórico que se ha desarrollado referente a la internacionalización de la educación superior ha tomado dos grandes visiones, una basada en la economía y la otra en la política. Desde la primera perspectiva se puede observar que el fenómeno de la internacionalización está directamente relacionado con la movilidad estudiantil, actividad que ha tenido suficientes intentos de medición por parte de la UNESCO, asimismo se han intentado hacer mediciones sobre los rendimientos económicos que trae consigo este fenómeno. En el aspecto político, la internacionalización se concibe a partir de las relaciones formales como un producto entre las instituciones de educación superior.

Coryell et al 2012, sugieren que existe la necesidad de tener una definición, concepto y/o entender lo mismo sobre la internacionalización de la educación superior, y sobre ¿cuáles deberían de ser o son los beneficios? de los estudiantes que participan en la movilidad internacional.

Por otro lado, algunas de las visiones sobre la internacionalización de la educación superior (IES), aluden que este fenómeno es resultado de las presiones globales (Brody, 2007) y que se expresan a partir de la capacidad de respuesta que tienen las instituciones (Knight, 2005,2004).

Si bien, existe el consenso generalizado sobre los elementos que la IES deben poseer para ser consideradas que están internacionalizadas, en la que principalmente sobresale el elemento de "la movilidad académica" y "la internacionalización del currículo" como los indicadores más relevantes. De Wit (2011), señala que una institución se ha internacionalizado, si se han añadido a esa gama indicadores como, los estudios internacionales, los estudios globales, la educación multicultural, la educación intercultural, educación para la paz. Posicionando en términos más extensos a la movilidad estudiantil, así como los estudios en el extranjero y la educación en el extranjero.

Es así, que la internacionalización se ha usado para identificar cambios internos en la educación superior, donde se sugiere que la integración de una dimensión internacional en la enseñanza, la investigación, y la prestación del servicio de la educación superior (Knigh y De Wit, 1995), continua siendo un eje central en la arena internacional de la educación superior.

También resulta pertinente resaltar que en términos de mercado la visión de Margison y van der Wende (2006), sobre la internacionalización de la educación superior, plantea que se debe asociar principalmente al posicionamiento que pueda tener una institución frente a sus competidores académicos, partiendo del hecho de que existe un consenso general sobre la necesidad que tienen las IES de hacer frente a las presiones globales. Dando con ello origen a la idea de una universidad de clase mundial.

En este sentido, es importante mencionar que actualmente la internacionalización se encuentra ante una crisis conceptual debido a dos serios cuestionamientos para la propia construcción del concepto, el primero y quizá más importante es 
¿Por qué razón las IES se deben de internacionalizar? Y el segundo es ¿Qué actividades y/o quienes le dirán a una IES se ha internacionalizado?, o ¿que está en buen camino para lograrlo?. Ante este escenario de Wit (2013) plantea que el contexto de la internacionalización se encuentra frente a cambios rápidos, por lo tanto se debe de reconceptualizar, ya que lo considera como una tautología de la educación internacional. Sin embargo, hay que puntualizar que para construir a la internacionalización de la educación superior, como concepto se deben tomar en cuenta las dimensiones a las que impacta en el momento de asumir que una IES se ha internacionalizado o bien algún programa se ha internacionalizado. A manera de ejemplo en el caso de Europa el programa de becas Erasmus Mundus que tiene como principal propósito hacer del espacio europeo un lugar de enseñanza de educación superior con calidad mundial donde puedan acceder estudiantes de países en vías de desarrollo, este programa ha servido de ejemplo al convertirse en un caso de éxito para generar otras prácticas y por lo tanto con una dimensión que va mas allá de lo internacional, en virtud de que en su inicio este programa estaba dirigido a estudiantes de América Latina, y ha sido de tal magnitud que su impacto e importancia, así como su evolución ha transitado hacia un programa donde tienen posibilidades de participar un estudiante de cualquier parte del mundo.

Referente a ¿Qué indicadores se deben tener en cuenta para considerar que una institución se ha internacionalizado o no? Actualmente la misma Knight (2011), (quien es precursora en estos estudios) argumenta la existencia de cinco mitos que persisten como indicadores en las instituciones, las cuales a modo de check list, han ido acumulando como logros pero que no necesariamente se debe considerar a esa institución que se ha internacionalizado, estos son: 1) Estudiantes extranjeros como agentes de internacionalización, 2) Reputación internacional como una forma de acercarse a la calidad, 3) Acuerdos institucionales internacionales, 4) Acreditación internacional y 5) Universidad de Marca Global.

$\mathrm{Si}$ bien estos elementos no contradicen la construcción del concepto de internacionalización, permiten explicar que la adopción de estos elementos queda a decisión de la institución que lo considere pertinente y que generalmente se presentan más en las instituciones de educación superior particulares que en las públicas.

Por otro lado, Haug (2010) sostiene que la autentica internacionalización de la educación superior posee elementos de tipo cultural, de gobernanza y de funcionamiento institucional. Estas son las siguientes:

a) La definición de la misión y de las estrategias institucionales en términos internacionales o «globales»; incluso universidades regionales que quieren realmente apoyar el desarrollo cultural, económico y social de su región.

b) La política institucional de reclutamiento de docentes y directivos: se orienta a lo local o a lo global dependiendo del propósito que quieran alcanzar.

c) La comunicación de la universidad por el desarrollo de una imagen institucional y una presencia internacional fuertes.

d) La educación transnacional, como resultado de la globalización de la educación superior.

Si bien, no es posible tener una definición exacta sobre la internacionalización de la educación superior y existe una dicotomía sobre los indicadores que se deben de considerar para su medición así como la dificultad para medir el alcance de este fenómeno tanto desde una perspectiva de externalidades positivas como de efectos negativos que pudiera tener, lo que no se puede poner en duda es que la internacionalización es considerada en la actualidad como un tema clave en el debate público de la educación superior. Es así que Brandenburg y De Wit (2011), argumentan que se debe repensar y redefinir la manera de asimilar a la internacionalización de la educación superior, debido a que ha sido percibida solamente como algo "bueno", mientras que la globalización como algo "malo". Lo cual le quita merito y pone de antagónico ambos conceptos, promoviendo una visión dicotómica y que no permite analiza los elementos de manera integral. En este sentido Teichler (2004) argumenta que el crecimiento económico, la igualdad de oportunidades, la mejora en la enseñanza y el desarrollo personal, la creación de vínculos entre la educación superior y el mercado laboral, la diversificación, cambios en la administración, la evaluación de la educación superior así como las tendencias hacia una sociedad del conocimiento, son políticas que se implementaron en Europa y que a partir de ello inició el debate de la internacionalización de la educación superior.

De manera similar este tipo de fenómenos se ha suscitado en países como en México, donde primero se llevó a cabo un proceso de federalización de la educación superior y posteriormente se consideró la dimensión internacional dentro del diseño de diversas políticas de educación superior. Pero que de manera paralela a las políticas federales las IES públicas mexicanas, sobre todo las fronterizas han aprovechado espacios en común produciendo buena prácticas de colaboración y cooperación académica (Navarro, 2012).

La idea principal, es que existe un vínculo entre la internacionalización y la globalización de la educación superior, y no precisa que uno sea "bueno" y el otro sea "malo", como ha sido señalado por Brandenburg y De Wit. Sino que la existencia de 
cada uno responde a la capacidad de asimilación institucional de las universidades sobre estos fenómenos y por lo tanto se observa una necesaria co-dependencia entre estos conceptos para explicarse.

Por lo tanto, la internacionalización de la educación superior es un fenómeno en construcción, que en términos muy prácticos ha contribuido en la búsqueda de elementos que permiten justificar la necesidad de creación y razón de ser de los vínculos formales que se producen entre IES. Esos vínculos, se hacen tangibles como resultado de diversas expresiones de internacionalización, dando con ello la eclosión de un conjunto de indicadores (movilidad estudiantil, académica, internacionalización del currículo, cambio en la estructura organizacional), los cuales implementados con las IES, se orientan a la búsqueda del ideal de la calidad de la enseñanza educativa, mediante el diseño de una política.

\section{Rasgos de internacionalización en las políticas de la educación superior en México}

Partiendo de que la internacionalización de la educación superior se asume como objeto de múltiples cambios asociados a la evolución del Estado en su acepción más abstracta. Los cuales se encuentran fijamente marcados por el fenómeno de la globalización, estos se han hecho cada vez más tangibles a través de la creación de estrategias por parte de las IES para el logro de la internacionalización de la educación superior. En esta parte del trabajo se abordan estos elementos desde una perspectiva de política pública, asociado principalmente a los mecanismos que en términos prácticos han servido para la toma de decisiones estatales haciéndose tangible u observables, a través del diseño e implementación de rasgos de la internacionalización en las políticas de educación superior en México.

Es por ello que se parte de la idea de que a través del impulso de la globalización se ha cambiado el contexto internacional teniendo efectos sobre la educación superior (Nayyar, 2008), percibiendo que existe una relación entre la globalización y la internacionalización teniendo como sustento y unidad de vínculo al Estado, siendo la educación superior, una actividad sustantiva monopolizada por este. Como se ha mencionado en el debate teórico, existen dos grandes visiones uno económico y otro en el aspecto político. Haciendo énfasis de que en el aspecto político la internacionalización se ha concebido a partir de la creación de vínculos formales.

Uno de los efectos en la educación superior derivado del fenómeno de la internacionalización, es que se considera como parte del proceso de adaptación, que supone un incremento en la facilidad para el intercambio de información, lo cual es permitido gracias al rápido desarrollo que ha tenido la tecnología (elementos necesarios para la comunicación), relacionado generalmente con el fenómeno de la globalización. Estas herramientas han facilitado la disminución drástica del tiempo que se invierte para contactarse entre actores, aun cuando estos estén situados en diferentes países (llamados nodos, desde un enfoque de "análisis de redes").

En este sentido, se retoma la responsabilidad para el Estado en cuanto al diseño eficiente y eficaz de políticas públicas que promuevan el rumbo y/o las directrices en materia de educación superior, mismas que repercuten directamente en las instituciones de educación superior (IES). Para ello el compartir experiencias entre instituciones ha sido un fenómeno que está asociado directamente con el concepto de internacionalización, pero motivado por la intencionalidad de compartir y generar mejores prácticas.

No obstante, en México la evolución de la política de educación superior está seriamente marcada por elementos contextuales internacionales, sin que ello haya implicado el diseño e implementación de una política específica para la internacionalización (Navarro, 2012), siendo hasta el sexenio foxista que se registra implícitamente como un eje central.

En el siguiente cuadro se muestra por sexenio, los lineamientos generales de la política implementada y el efecto materializado en el cambio institucional, que se ha plasmado a través de diferentes mecanismos. 
Tabla 1. Políticas de educación superior por periodo sexenal en México 1982-2012.

\begin{tabular}{|c|c|c|c|}
\hline Periodo & Presidente & Política & Cambio institucional \\
\hline $1982-1988$ & $\begin{array}{l}\text { Miguel de la } \\
\text { Madrid }\end{array}$ & $\begin{array}{l}\text {-La evaluación de la educación } \\
\text { superior en México, } 1984 \text {. }\end{array}$ & $\begin{array}{l}\text { Se enfatizaban los controles de calidad y eficiencia } \\
\text { para acceder a nuevas formas de financiamiento. }\end{array}$ \\
\hline 1988-1994 & $\begin{array}{l}\text { Carlos } \\
\text { Salinas de } \\
\text { Gortari }\end{array}$ & $\begin{array}{l}\text {-Aportaciones de la ANUIES para la } \\
\text { educación Superior, } 1989 . \\
\text {-Se crea la CONAEVA en } 1989 . \\
\text {-Propuestas de Lineamientos para } \\
\text { la evaluación de la educación } \\
\text { Superior. }\end{array}$ & $\begin{array}{l}1994 \text { La internacionalización. Con motivo del Tratado } \\
\text { de Libre Comercio de Norteamérica (México, Estados } \\
\text { Unidos y Canadá) } \\
\text { Practicas formales a partir del establecimiento de } \\
\text { convenios. }\end{array}$ \\
\hline 1994-2000 & $\begin{array}{l}\text { Ernesto } \\
\text { Zedillo Ponce } \\
\text { de León }\end{array}$ & $\begin{array}{l}\text {-Crecimiento de Universidades } \\
\text { Tecnológicas, de la matricula de } \\
\text {-Crecimiento de } \\
\text { posgrado. } \\
\text {-Continuidad al Programa de } \\
\text { Mejoramiento del Profesorado }\end{array}$ & $\begin{array}{l}\text { En } 1994 \text { fue creado el Programa Nacional de } \\
\text { Superación del Personal Académico, que otorga becas } \\
\text { para académicos en servicio que deseen realizar } \\
\text { estudios de posgrado. } \\
\text { Creación de CENEVAL con el objeto de fortalecer la } \\
\text { calidad educativa. } \\
\text { Creación e implementación del programa PRONAD. }\end{array}$ \\
\hline $2000-2006$ & $\begin{array}{l}\text { Vicente Fox } \\
\text { Quezada }\end{array}$ & $\begin{array}{l}\text { Desarrollo Social y humano con } \\
\text { énfasis en educación de vanguardia. }\end{array}$ & $\begin{array}{l}\text { Participación y colaboración con organismos } \\
\text { internacionales para la realización de estudios } \\
\text { comparados. }\end{array}$ \\
\hline 2006-2012 & $\begin{array}{l}\text { Felipe } \\
\text { Calderón } \\
\text { Hinojosa }\end{array}$ & $\begin{array}{l}\text { Crecimiento de Instituciones de } \\
\text { Educación Superior }\end{array}$ & $\begin{array}{l}\text { Búsqueda de la calidad de competencia } \\
\text { internacional en todos los niveles de la educación } \\
\text { superior. } \\
\text { Recomendaciones OECD. }\end{array}$ \\
\hline $2013-2018$ & $\begin{array}{l}\text { Enrique Peña } \\
\text { Nieto }\end{array}$ & $\begin{array}{l}\text { La estrategia: "México } \\
\text { educación de Calidad" }\end{array}$ & $\begin{array}{l}\text {-Sistema de profesionalización docente, } \\
\text { fundamentado en la evaluación. } \\
\text {-Promoción de dominio de TIC's. } \\
\text {-Crear un programa de estadías de estudiantes y } \\
\text { profesores en instituciones extranjeras de } \\
\text { educación superior. }\end{array}$ \\
\hline
\end{tabular}

Fuente: Elaboración propia en base a información de los planes nacionales de desarrollo, los informes de gobierno de México, Planes Nacionales de Desarrollo y Luengo (2003), disponible en:

http://www.anuies.mx/e proyectos/pdf/04 Las reformas en la Educacion Superior en Mexico.pdf y Brunner (1994), disponible en: http://bibliotecavirtual.clacso.org.ar/ar/libros/argentina/cedes/brunner.rtf.

Esta tabla muestra cual ha sido en terminos generales la orientación de las politicas de educacioón superior en México y la adopción de los principales rasgos caracteristicos.

En el marco de las políticas federales de educación, se observa que a partir de la toma de conciencia institucional, la adopción de rasgos internacionales en las políticas de Estado han sido tardías, en contraste con el marco global de educación superior tomando como referente la primera conferencia mundial de educación superior celebrada en Paris en 1998, donde se reflexiona profundamente sobre la necesidad de promover la internacionalización, como una estrategia para generar la paz mundial partiendo de valores fundamentales, principalmente la tolerancia a otras culturas.

Sin embargo, en el sexenio de Felipe Calderón persiste la necesidad por la búsqueda de la calidad, lo cual responde a los lineamientos internacionales que dictan las políticas de educación.

Por otro lado, vale la pena resaltar que se percibe un rompimiento en la continuidad de la política, en lo que va del sexenio de Felipe Calderón al actual presidente Enrique Peña Nieto, ya que mientras Calderón buscaba la competencia internacional a través de la adopción de las recomendaciones de la OECD, el actual presidente, se percibe más modesto al buscar implementar un programa de movilidad académica y estudiantil, práctica ya recurrente e institucionalizada en las diferentes IES de México. Ya que textualmente en el actual Programa Sectorial de Educación, 2013-2018, en la estrategia 2.3 del objetivo 2 , la cual dice "Continuar el desarrollo de los mecanismos para el aseguramiento de la calidad de los programas $e$ instituciones de educación superior", se ha propuesto en la línea de acción 2.3.8. "Apoyar nuevos modelos de cooperación académica para la internacionalización de la educación superior" (PSE, 2013-2018).

Lo cual sugiere que se acepta la necesidad de dejar implícita en una política de gobierno que las prácticas de internacionalización de la educación superior, le deberá conducir a la calidad, reconociendo que existen esfuerzos precedentes a esta política, los cuales han sido el principal impulso y reconocimiento que deben y pueden tener las IES al mostrarse como ejemplos de éxito en sus procesos internos de internacionalización. 


\section{Implementación y asimilación para las instituciones de educación superior en la región noroeste de México}

Este proceso es resultado de la capacidad de respuesta que cada IES desarrolla e implementa en virtud de que los cambios estructurales en ellos no han sido simultáneos, ni siquiera paralelos, de ahí que han implementado algún tipo de programa para la movilidad estudiantil, que no necesariamente está acompañado de una normatividad que regule las actividades en torno a ello, dejando a discreción el proceso en términos tanto académicos como administrativos.

Partiendo de este fenómeno es de gran importancia resaltar lo que Lahera (2004), dice sobre que "las políticas públicas sin política tienen un problema de diseño", debido a que se observó en términos generales que en sus modelos educativos no se plantea como un eje central la internacionalización, surgiendo con ello una postura contrapuesta internamente en la propia institución, en el sentido de que administrativamente se le dedican esfuerzos a las actividades de movilidad estudiantil y /o académica, observándose que en su mayoría las Universidades Públicas (UPE'S) de la región noroeste de la ANUEIS, no han definido un marco regulatorio sino sólo lineamientos institucionales para su operatividad. En el caso de la UJED, destaca el hecho, de que en su modelo educativo se planteen a la internacionalización como un aspecto relevante, no obstante, recientemente diseñaron un reglamento de movilidad estudiantil y académica, pero las actividades son absorbidas por una dirección de planeación y desarrollo académico, lo cual por su nombre sugiere la práctica de otro tipo de funciones.

Otro caso que merece mención aparte, es el de UABC, ya que en su modelo educativo no está considerada la internalización como un eje central (aunque considera a la movilidad en términos generales), aunado a que no existe un reglamento explícito que regule las actividades sino sólo lineamientos internos (dejando a discreción la selección para su participación), esto en contraposición al hecho de que dediquen esfuerzos administrativos y financieros para la internacionalización al contar con una estructura que oriente dichas actividades, denominada "Coordinación de Cooperación Internacional e Intercambio Académico" que opera el programa de movilidad estudiantil, diseñado para cumplir con esas funciones, así como el diseño e implementación del "Proyecto UABC Internacional”, del actual periodo rectoral ${ }^{1}$.

\footnotetext{
1 Respecto al caso de la Universidad Autónoma de Baja California, vale la pena destacar que durante la actual administración (2015-2019), el tema de la política para su internacionalización ha tomado mayor relevancia en términos
}

Por otro lado, instituciones como UACJ, que cuenta con un reglamento pero no con un programa explícito, viene a recordar lo que Lindblom (1992) aportó en el ámbito de políticas públicas referente a la clásica idea de "las políticas para salir del paso", así como lo que Lahera (2004) afirma sobre que las políticas sin política pública son pura demagogia, esto con el ánimo de acentuar que las IES actúan presionadas por la demanda social de un mejor servicio más que por el diseño institucional en el marco de una política federal. discursivos, ya que en el nuevo modelo educativo se considera a la movilidad o intercambio como un componente de la etapa disciplinaria o terminal. También cuenta con dos versiones de modelo educativo versión 2006 y versión 2013. 
Tabla 2. Elementos organizacionales que las IES de la región noroeste han implementado.

\begin{tabular}{|c|c|c|c|c|c|}
\hline & Institución & $\begin{array}{ll}\text { Programa } & \text { de } \\
\text { Movilidad } & \\
\end{array}$ & Cambio Normativo* & Cambio Organizacional & $\begin{array}{l}\text { Modelo } \\
\text { Educativo } \\
\end{array}$ \\
\hline 1 & $\begin{array}{l}\text { Universidad } \\
\text { Autónoma de Baja } \\
\text { California }\end{array}$ & $\begin{array}{l}\text { Programa de } \\
\text { Movilidad Estudiantil }\end{array}$ & No hay reglamento & $\begin{array}{l}\text { Coordinación de Cooperación } \\
\text { Internacional e Intercambio } \\
\text { Académico }\end{array}$ & No \\
\hline 2 & $\begin{array}{l}\text { Universidad } \\
\text { Autónoma de Baja } \\
\text { California Sur }\end{array}$ & Movilidad Estudiantil & No hay reglamento & $\begin{array}{l}\text { No cuentan con un órgano } \\
\text { encargado de esas actividades } \\
\text { de forma exclusiva } \\
\text { Departamento de Servicios } \\
\text { Estudiantiles }\end{array}$ & No \\
\hline 3 & $\begin{array}{l}\text { Universidad } \\
\text { Autónoma de } \\
\text { Chihuahua }\end{array}$ & $\begin{array}{l}\text { Programa ECOES Y } \\
\text { Programa de } \\
\text { Movilidad en conjunto } \\
\text { con ANUIES }\end{array}$ & No hay reglamento & $\begin{array}{l}\text { Departamento de Movilidad y } \\
\text { atención a Estudiantes }\end{array}$ & No \\
\hline 4 & $\begin{array}{l}\text { Universidad } \\
\text { Autónoma de } \\
\text { Ciudad Juárez } \\
\text { (UACJ) }\end{array}$ & $\begin{array}{l}\text { No cuenta con un } \\
\text { programa pero } \\
\text { "Actividades } \\
\text { relacionadas con la } \\
\text { movilidad Estudiantil" }\end{array}$ & $\begin{array}{l}\text { Reglamento de } \\
\text { Movilidad Estudiantil }\end{array}$ & $\begin{array}{l}\text {-Dirección General de } \\
\text { Vinculación e Intercambio } \\
\text {-Subdirección de Movilidad } \\
\text { Estudiantil }\end{array}$ & No \\
\hline 5 & $\begin{array}{l}\text { Universidad } \\
\text { Juárez del Estado } \\
\text { de Durango } \\
\text { (UJED) }\end{array}$ & $\begin{array}{l}\text { "Estancias de } \\
\text { Movilidad" }\end{array}$ & $\begin{array}{l}\text { Reglamento de } \\
\text { Movilidad Estudiantil } \\
\text { y Académica }\end{array}$ & $\begin{array}{l}\text { Dirección de Planeación y } \\
\text { Desarrollo Académico }\end{array}$ & $\mathrm{Si}$ \\
\hline 6 & $\begin{array}{l}\text { Universidad } \\
\text { Autónoma de } \\
\text { Sinaloa (UAS) } \\
\end{array}$ & $\begin{array}{l}\text { Programa de } \\
\text { Movilidad Estudiantil }\end{array}$ & $\begin{array}{l}\text { Reglamento de } \\
\text { Intercambio y } \\
\text { Movilidad Estudiantil } \\
\end{array}$ & $\begin{array}{l}\text { Dirección General de } \\
\text { Vinculación y Relaciones } \\
\text { Internacionales }\end{array}$ & No \\
\hline 7 & $\begin{array}{l}\text { Universidad de } \\
\text { Sonora (UNISON) }\end{array}$ & $\begin{array}{l}\text { "Actividades } \\
\text { Movilidad } \\
\text { Alumnos" }\end{array}$ & $\begin{array}{l}\text { Reglamento de } \\
\text { Movilidad de Alumnos }\end{array}$ & $\begin{array}{lll}\text { Dirección de } & \text { Vinculación } & \text { y } \\
\text { Difusión } & & \\
\text {-Dirección de } & \text { Movilidad } & \text { y } \\
\text { Cooperación Académica } & \end{array}$ & No \\
\hline
\end{tabular}

Fuente: Elaboración propia, con información de las páginas web oficiales de las Instituciones mencionadas.

En este contexto será pertinente reflexionar ¿Cuál ha sido el rumbo de la internacionalización de la educación superior en la región noroeste?, así como el hecho de que en ausencia de una política federal explicita que guíe las actividades para la internacionalización de la educación superior en México, pone de manifiesto que el éxito de una institución de Educación Superior únicamente dependerá de sus propios esfuerzos y el desarrollo de sus propias capacidades para crear mecanismos innovadores que le permitan elevar su calidad de este servicio público.

Por otro lado, existen elementos y esfuerzos por parte del mismo gobierno federal para incentivar la cooperación binacional, lo cual se ha entendido como una extensión que se deriva del fenómeno de la internacionalización en materia de educación superior (Montano, Mungaray y Galaz, 2008, en Navarro y Moctezuma, 2012), aun cuando persiste la ausencia de una política pública y de un presupuesto explicito que lo promueva de manera directa para la búsqueda de la calidad.

\section{Conclusión}

Las diversas aportaciones para la construcción de diversas opiniones referentes a la internacionalización de la educación superior han permitido adquirir una perspectiva de cambio y adaptación. En el sentido de que si bien existen coincidencias en cuanto que elementos se consideran van a internacionalizar a una IES, en su mayoría las UPE'S de la región noroeste operan en términos conceptuales con rasgos que Knight denomina como mitos, por lo que son necesarios que ocurran dos cosas, la primera que se llegue a un conceso referente a cómo definir los indicadores y/o rasgos para la aceptación de la internacionalización y la segunda que las IES se autodefinan en función de una política construida a largo plazo.

También se han presentado serios cuestionamientos sobre estos elementos, motivando la generación de un debate en torno a ¿cuáles son los indicadores que internacionalizan a las universidades?, ¿cuándo se ha conseguido la meta de internacionalizarse?, asimismo ¿quién dice que ha logrado internacionalizarse?, y yendo un poco más allá ¿por cuánto tiempo se internacionaliza una institución?. En este sentido vale la pena señalar que la proliferación de órganos de dimensión internacional ha contribuido a la generación de esta confusión. Esta situación no debe demeritar el trabajo y esfuerzos que cada IE realiza en virtud de que responden a realidades 
diferentes condicionadas por sus contextos socioeconómicos y políticos.

En México, los rasgos de internacionalización de la educación superior han sido más visibles en la política a partir del periodo foxista, tomando en cuenta que queda implícita como un rasgo de la misma, sin embargo, ello no sugiere que los esfuerzos previos no sean significativos, por lo que, los primeros indicios de la internacionalización son percibidos a partir de la necesidad de la evaluación por la calidad y la eficiencia, en un sentido meramente administrativo, pero transitando hacia el establecimiento de un marco formal-legal.

Esto último, ha permitido la creación de lazos a través de la firma de convenios entre IES para compartir experiencias institucionales en la búsqueda de la riqueza y de las mejores prácticas, así como en algunos casos la autorregulación de estas prácticas mediante un reglamento interno o bien la creación de lineamientos generales para orientar la homogenización de estas actividades, que conlleve a la homogenización de procesos entre instituciones y equilibrio entre criterios.

En lo que respecta la UPE's de la región noroeste de la ANUIES, se debe reconocer que los diferentes mecanismos instrumentados para la operación e implementación de sus respectivos programas de movilidad estudiantil y /o académica, son producto de sus esfuerzos diferenciados por las condiciones y capacidades tanto financieras como administrativas, las cuales están plasmadas en esos rasgos.

Es así que la internacionalización de la educación superior en México se percibe a través de la generación de políticas en las universidades públicas estatales, las cuales en un sentido social deberán ser producto de la creación y reconocimiento de un espacio propicio para el desarrollo de mejores prácticas y la emulación de estas, dejando como principal interrogante: ¿cuál será el rumbo de la política de internacionalización que se le dará a partir de la línea de acción planteada en el programa sectorial educativo 20132018 y en períodos futuros? 


\section{Referencias}

De Wit, H. (2011). Globalización e internacionalización de la educación superior. Revista de Universidad y Sociedad del Conocimiento (RUSC). Vol. 8 No 2, págs. 77-84. UOC.

Guerrero, O. (2000). Administración Pública. Léxico de Política, FCYP-UNAM, UAM, FLACSO, México.

Haug, G. (2010). La internacionalización de la educación superior: más allá de la movilidad europea. La cuestión Universitaria, \#6.

Held, D. et al. (2000), Rethinking Globalization. In Held, D. \& McGrew, A. (2000) The Global Transformations Reader; Cambridge: Polity Press.

Hobbes, T. (1980). Leviatán. México, Fondo de Cultura Económica. Original de (1651)

Knight, J. (2005). An Internationalization Model: Responding to New Realities and Challenges: The International Bank for Reconstruction and Development. In De Wit et al (eds.) The World Bank. Higher Education in Latin America. Washington D.C. The World Bank.

Knight, J. (2011). Cinco Mitos sobre la Internacionalización. Educación Superior Internacional. No. 62. Páginas 1415.

Lahera P., E. (2004). Política y Políticas Públicas. División de Desarrollo Social, CEPAL, Políticas Sociales. Serie 95.

Lindblom, C. (1992). La Ciencia de Salir del Paso. En Lowi, T. J. La hechura de las políticas. L. F. A. Villanueva (eds.). Miguel Ángel Porrúa.

Luengo, E. (2003), Tendencias De La Educación Superior En México: Una Lectura Desde La Perspectiva de la Complejidad. Trabajo elaborado para el Seminario sobre Reformas de la Educación Superior en América Latina y el Caribe, realizado el 5 y 6 de junio del 2003, en Bogotá, Colombia, bajo los auspicios del Instituto Internacional de la UNESCO para la Educación Superior en América Latina y el Caribe (IESACC) y la Asociación Colombiana de Universidades (ASCUN). http://www.anuies.mx/e_proyectos/pdf/04_Las_reformas_en_la_Educacion_Superior_en_Mexico.pdf

Marginson, S. y van Der Wende, M. (2007). Globalisation and Higher Education. Working Papers, No. 8, OECD Publishing. http://dx.doi.org/10.1787/173831738240

Navarro C., A.y Moctezuma, P. (2012). La Globalización de la Administración Pública en la Educación Superior Mexicana. México. Universidad Autónoma de Baja California.

Nayyar, D. (2008). Globalization and Markets: Challenges for Higher Education. Higher Education in the World. http://upcommons.upc.edu/revistes/bitstream/2099/8115/1/nayyar.pdf

OECD (2009). Globalization and Higher Education: What might the future bring?. Program on Institutional Management in Higher Education. December.

Plan Nacional de Desarrollo 2013-2018. Sexenio a cargo del Presidente Enrique Peña Nieto.

Programa Sectorial Educativo 2013-2018. Sexenio a cargo del Presidente Enrique Peña Nieto.

ERASMUS MUNDUS, Pagina web oficial del Programa: http://eacea.ec.europa.eu/erasmus_mundus/programme/who_participate_en.php

Universidad Autónoma de Baja California. (2016). Uabc.mx. Recuperado el día 05 de Febrero de 2016, de http://www.uabc.mx/formacionbasica/modeloedu.htm

Universidad Autónoma de Baja California Sur. (2016). Uabcs.mx. Recuperado el día 05 de Febrero de 2016, de http://www.uabcs.mx/secciones/contenido/15

Universidad Autónoma de Baja California Sur. (2016). Uabcs.mx. Recuperado el día 04 de Febrero de 2016, de http://uabcs.mx/secciones/contenido_pagina/279/795/Direcci\%C3\%B3n\%20de\%20Docencia\%20e\% 20Investigaci\%C3\%B3n\%20Educativa

Universidad Autónoma de Chihuahua. (2016). Uach.mx. Recuperado el día 05 de Febrero de 2016, de http://www.uach.mx/academica y escolar/modelo educativo/2008/05/21/modelo educativo uach/

Universidad Autónoma de Chihuahua. (2016). Uach.mx. Recuperado el día 10 de Febrero de 2016, de http://www.uach.mx/planeacion/2010/08/09/7informe.pdf

Universidad Autónoma de Ciudad Juárez. (2016). Uacj.mx. Recuperado el día 10 de Febrero de 2016, de http://www.uacj.mx/sa/ie/Documents/Certificaci\%C3\%B3n\%20PIMECIME/Docentes/Modelo Educati vo UACJ.pdf

Universidad Autónoma de Ciudad Juárez. (2016). Uacj.mx. Recuperado el día 11 de Febrero, de http://www.uacj.mx/Paginas/Default.aspx

Universidad Juárez del Estado de Durango. (2016). Ujed.mx. Recuperado el día 11 de Febrero de 2016, de http://www.ujed.mx/portal/publico/inicio.aspx

Universidad Juárez del Estado de Durango. (2016). Ujed.mx. Recuperado el día 09 de Marzo de 2016, de http://www.ujed.mx/portal/Publico/Escuela0 Documentacion.aspx

Universidad Autónoma de Sinaloa. (2016). Uas.edu.mx. Recuperado el día 11 de Febrero de 2016, de http://www.uas.edu.mx/web/

Universidad de Sonora. (2016). Uson.mx. Recuperado el día 10 de Febrero de 2016, de

http://www.uson.mx/ 
Revista Internacional de Aprendizaje en Educación Superior, 4(2), 2017, pp. 71-80

http://www.uabc.mx/formacionbasica/modeloedu.htm

http://www.uabcs.mx/secciones/contenido/15

http://uabcs.mx/secciones/contenido pagina/279/795/Direcci\%C3\%B3n\%20de\%20Docencia\%20e\%20

Investigaci\%C3\%B3n\%20Educativa

http://www.uach.mx/academica_y_escolar/modelo_educativo/2008/05/21/modelo_educativo_uach/

http://www.uacj.mx/sa/ie/Documents/Certificaci\%C3\%B3n\%20PIMECIME/Docentes/Modelo_Edu cativo_UACJ.pdf

http://www.uas.edu.mx/web/

http://www.uson.mx/

http://www.uacj.mx/Paginas/Default.aspx

http://www.ujed.mx/portal/publico/inicio.aspx

http://www.uach.mx/planeacion/2010/08/09/7informe.pdf 